\title{
Pill-ID: Matching and Retrieval of Drug Pill Images
}

\author{
Young-Beom Lee ${ }^{\mathrm{a}}$, Unsang Park ${ }^{\mathrm{b}}$, Anil K. Jain ${ }^{\mathrm{a}, \mathrm{b}, *}$, Seong-Whan Lee ${ }^{\mathrm{a}}$ \\ ${ }^{a}$ Department of Brain and Cognitive Engineering, Korea University, Anam-dong, Seongbuk-gu, Seoul 136-713, Korea \\ ${ }^{b}$ Department of Computer Science and Engineering, Michigan State University, E. Lansing, MI 48824, USA
}

\begin{abstract}
Worldwide, law enforcement agencies are encountering a substantial increase in the number of illicit drug pills being circulated in our society. Identifying the source and manufacturer of these illicit drugs will help deter drug-related crimes. We have developed an automatic system, called Pill-ID to match drug pill images based on several features (i.e., imprint, color, and shape) of the tablet. The color and shape information is encoded as a three-dimensional histogram and invariant moments, respectively. The imprint on the pill is encoded as feature vectors derived from SIFT and MLBP descriptors. Experimental results using a database of drug pill images (1,029 illicit drug pill images and 14,002 legal drug pill images) show 73.04\% (84.47\%) rank-one (rank-20) retrieval accuracy.
\end{abstract}

Keywords: Image retrieval, pill images, illicit drugs, imprints, moment invariants, color histogram

\section{Introduction}

Illicit drugs, widely circulated in the international market, are one of the major factors influencing criminal activities. They also lead to additional enforcement and tracking expense for law enforcement units. More than 35 million individuals either used illicit drugs or abused prescription drugs in the United States in 2007 alone. The U.S. federal government allocated more than $\$ 14$ billion for drug treatment and prevention, counterdrug law enforcement, drug interdiction and international counter-drug assistance in 2009i . Drug trafficking is also one of the major factors behind violent and other illegal activities ${ }^{\mathrm{ii}}$.

U.S. Drug Enforcement Administration's (DEA) Office of Forensic Sciences has been publishing Microgram Bulletin and Microgram Journal to assist forensic scientists for detection and analysis of drug-related substances $^{\text {iii }}$. Food and Drug Administration (FDA) regulations $^{\text {iv }}$ require that every prescription pill or capsule sold in the market must have unique look for easy identification in terms of size, shape, color, and imprint. An imprint is an indented or printed mark on a pill, tablet,

\footnotetext{
*Corresponding author, Tel.:+1 5173559282.

Email addresses: young-beom_lee@korea.ac.kr (Young-Beom Lee), parkunsa@cse.msu.edu (Unsang Park), jain@cse.msu.edu (Anil K. Jain ), swlee@image.korea.ac.kr (Seong-Whan Lee)

${ }^{1}$ An earlier version of this work appeared in (Lee et al., 2010).
}

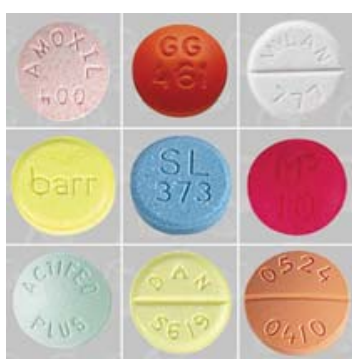

(a)

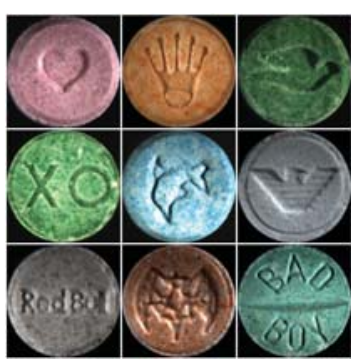

(b)
Figure 1: Example drug pill images. (a) Legal drug pills and (b) illicit drug pills.

or capsule. Imprints can be a symbol, text, a set of digits, or any combination of them ${ }^{\mathrm{v}}$.

Illicit drug makers use imprints, color, and shape to identify the chemical substances and their quantities in each pill. Special imprints on the pills are also used for advertisement purposes. When a new illicit psychoactive pill is first detected in the market, its information is recorded in the law enforcement databases ${ }^{\mathrm{vi}}$. This information includes chemical and physical description, where the physical description includes shape, color, imprint, etc. Fig. 1 shows example images of legal and illicit drug pills.

Law enforcement units would like to automatically extract the information about an illicit drug pill (i.e., type of pill, manufacturing location, and the manufacturer) by matching its image with known patterns in 


\begin{tabular}{c|c|c|c}
\hline Image & ot & & \\
\hline $\begin{array}{c}\text { Product } \\
\text { No. }\end{array}$ & 001 & 002 & 003 \\
\hline Form & TABLET & TABLET & TABLET \\
\hline Dosage & Oral & Oral & $\begin{array}{c}\text { Oral } \\
\text { 100MG }\end{array}$ \\
\hline $\begin{array}{c}\text { Drug } \\
\text { name }\end{array}$ & $\begin{array}{c}\text { CAPO } \\
\text { TEN }\end{array}$ & $\begin{array}{c}\text { CAPO } \\
\text { TEN }\end{array}$ & $\begin{array}{c}\text { CAPO } \\
\text { TEN }\end{array}$ \\
\hline $\begin{array}{c}\text { Active } \\
\text { ingredient }\end{array}$ & $\begin{array}{c}\text { CAPTO } \\
\text { PRIL }\end{array}$ & $\begin{array}{c}\text { CAPTO } \\
\text { PRIL }\end{array}$ & $\begin{array}{c}\text { CAPTO } \\
\text { PRIL }\end{array}$ \\
\hline
\end{tabular}

Figure 2: Example legal pill images and the associated information in the FDA database.

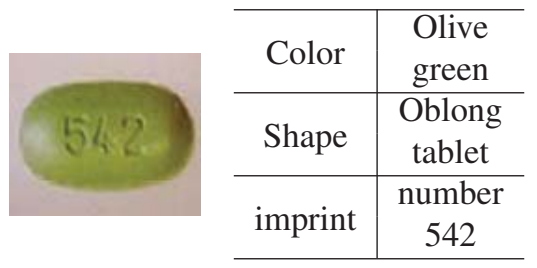

Figure 3: Image of the Rohypnol pill.

their databases. Legal drug pills can be identified from the information provided by pharmaceutical companies enrolled in the FDA database. Fig. 2 shows the FDA information of three legal pills of the same type which have different dosage.

Unlike legal drug pills, illicit drug pills do not follow any regulation about their distinctiveness for the purpose of identification. Fig. 3 shows an image of an addictive illicit drug pill, called "Rohypnol" and its attributes. Information in the DEA database indicates that Rohypnol is currently supplied with a 1-milligram dose in an olive green, oblong tablet, imprinted with the number 542. In general, matching pill images with the patterns of previously seized pills is the only effective way to identify illicit drug pills.

Consequently, it is important to develop an image based matching tool to automatically identify illicit drug pills based on their imprint, size, shape, color, etc. There are a few web sites that provide keyword-based legal drug pill identification tools $\mathrm{s}^{\mathrm{v} \text { vii }}$. The keywords are based on the size, shape, and color of the pill (e.g., round, diamond, red, etc.), but they do not utilize the imprint. Keyword-based retrieval has a number of known limitations, namely keywords are subjective and do not capture all the information about the pill for accurate retrieval.

To develop a successful automatic pill image match- ing system, it is important to compensate for the variations in the appearance of the pills, due, for instance, to changes in viewpoint, illumination or occlusion (Riesenhuber and Poggio, 2000). For this reason, we utilize the gradient magnitude information to characterize the imprint patterns on the drug pill images.

Gradient magnitude is more stable than color or gray scale especially against illumination variations. Given the gradient magnitude image, Scale Invariant Feature Transform (SIFT) descriptor and Multi-scale Local Binary Pattern (MLBP) descriptors are used to generate feature vectors. In addition, invariant moment features proposed by $\mathrm{Hu}(\mathrm{Hu}, 1962)$ and color histogram are used to generate shape and color feature vectors, respectively. With the extracted feature vectors, we use the $L_{2}$-norm to compute the similarity between two pill images. The proposed retrieval system, called Pill-ID is evaluated on a database consisting of 1,029 illicit drug pill images and 14,002 legal drug pill images.

The rest of the paper is organized as follows. Section 2 discusses related work, section 3 describes feature extraction and matching scheme, section 4 presents experimental results, and section 5 concludes the paper.

\section{Related Work}

Given a query image, the goal of Content Based Image Retrieval (CBIR) is to retrieve visually similar images from a large image database on the basis of low level image features (such as color, texture, and shape). Constituents of the image features are extracted from both query and gallery images and used to find gallery images which are most similar to the query. Contrary to the keyword based image retrieval, CBIR does not need any manual labeling of the query image. One of the seminal paper on CBIR is by Chang et al. (Chang and Liu, 1984), who proposed a picture indexing and abstraction method. To construct picture indices, abstraction operations were formulated to perform picture object clustering and classification.

The terminology CBIR was first introduced in the literature in (Kato, 1992), where automatic retrieval of images from a database based on color and shape features was proposed. QBIC was the first functional CBIR system reported (Flickner et al., 1995). QBIC allowed image retrieval using user-constructed sketches and drawings, selected colors and texture patterns, or other graphical information as queries. In spite of extensive research and development in image retrieval, the performance of general purpose CBIR systems is less than desired. As a result, the focus in CBIR has now 


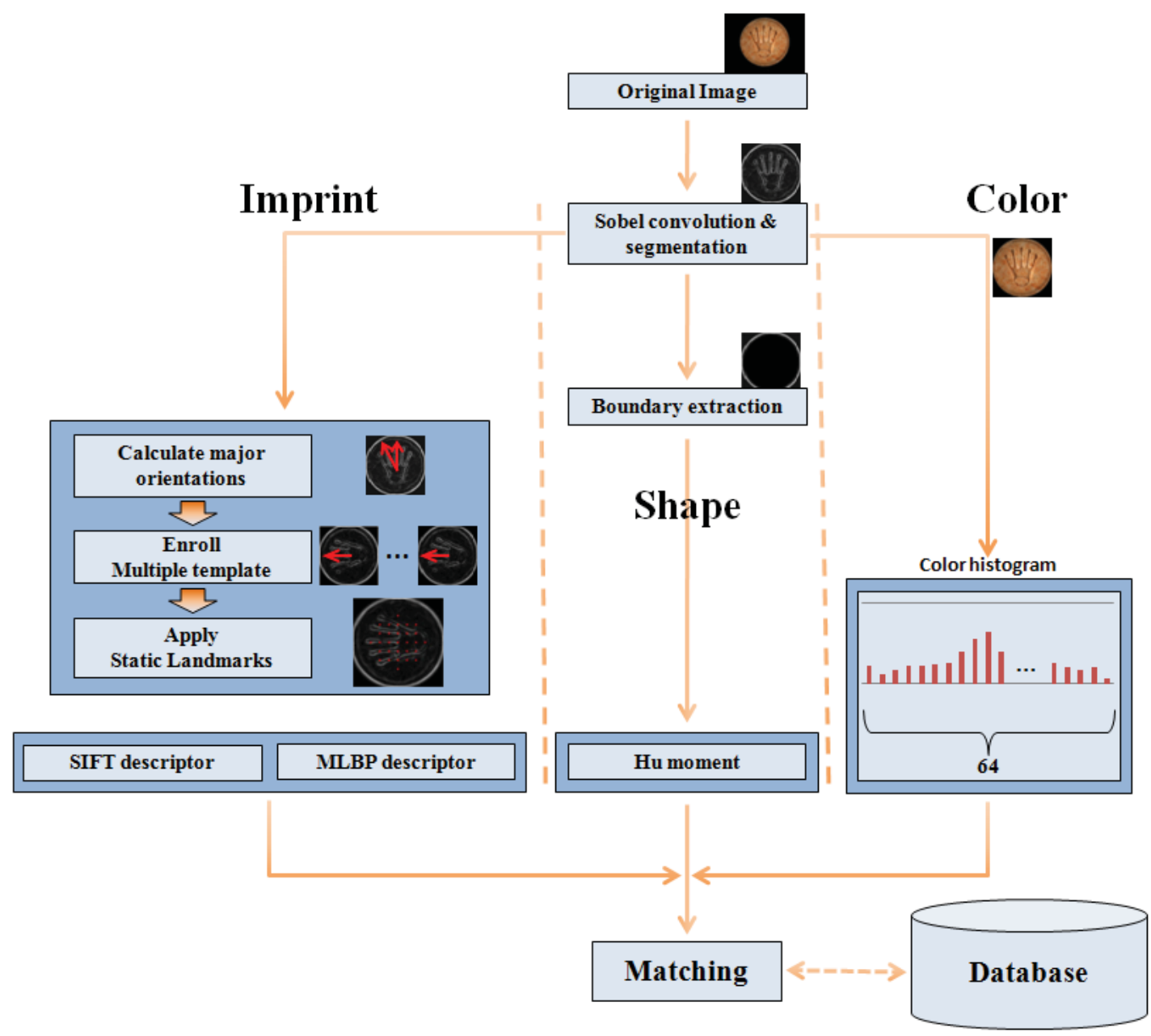

Figure 4: Schematic of the Pill-ID system.

shifted towards special purpose (domain-specific) image retrieval with the goal of finding "near duplicate" images. One such and successful CBIR system for law enforcement applications is the Tattoo-ID system developed by Jain et al. (Jain et al., 2009). This system automatically extracts features from tattoo images based on the Scale Invariant Feature Transform (SIFT) to find near duplicate tattoos in a large database of tattoos.

There have been only a few studies on pill image matching and retrieval. Geradts et al. (Geradts and Bijhold, 2002) used shape features, including size of the bounding box, density inside the bounding box, and contour of imprints. To decouple the variants of translation, rotation, and scale, images were first translated from orthogonal coordinates to the log polar coordinates, and then the two-dimensional cross-correlation function was used for matching log polar data. They chose only three different pills (out of 432 pill images) as the query set and synthetically generated 75 images by rotating those three pills at 25 different angles. Given the 75 queries (only three distinct imprint patterns) with a gallery database of 432 pill images, the authors reported a $100 \%$ matching accuracy.

\section{Feature Extraction \& Matching}

We use the gradient magnitude image in segmentation and feature vector construction for the shape and imprint of a pill. Feature vectors for imprints, color, and shape are constructed based on distribution based descriptors (i.e., SIFT and MLBP), color histogram, and $\mathrm{Hu}$ moments, respectively. The overall process is shown in Fig. 4. 


\subsection{Preprocessing}

Input pill image is first smoothed with a Gaussian filter, followed by a convolution operation using the Sobel operator (Gonzalez and Woods, 1992) to generate the gradient magnitude image. Given the original image $I(x, y)$, the gradient magnitude image $M(x, y)$ is obtained as

$$
M(x, y)=S_{x} * I(x, y)+S_{y} * I(x, y)
$$

where $S_{x}$ and $S_{y}$ are Sobel operators in $x$ and $y$ directions, respectively and ' $*$ ' represents the convolution operator. The segmentation process is straightforward using $M(x, y)$ due to the relatively uniform background color in most of the pill images (see Fig. 4). After tightly cropping the pill image, the image size is normalized to $200 \times 200$ pixels. Fig. 5 shows color, gray scale, and gradient magnitude images. The gradient magnitude image has smaller intra-class variations compared to color or gray scale images. The feature extraction methods (i.g., SIFT and MLBP) also provide better matching accuracy when they were applied to the gradient magnitude images compared to gray scale images.

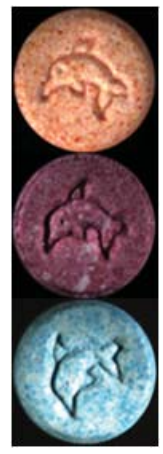

(a)

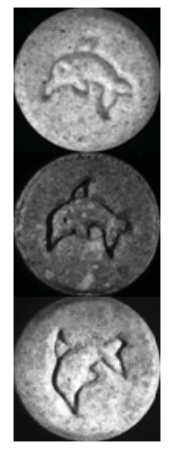

(b)

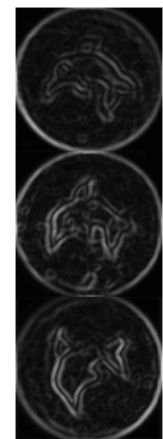

(c)
Figure 5: Color, gray scale, and gradient magnitude images. (a) input color image, (b) gray scale image, and (c) gradient magnitude image.

\subsection{Imprint descriptor construction}

\subsubsection{SIFT descriptor}

Scale Invariant Feature Transform (SIFT) (Lowe, 2004) is one of the most popular local representation schemes used for object recognition and CBIR. Computation of SIFT features consists of two stages: (i) keypoint extraction, and (ii) descriptor calculation in a local neighborhood at each keypoint. In our application, for computational efficiency, we place a regular grid of landmarks within a preset radius on the pill image instead of extracting keypoints. A set of keypoints
(=29) are selected within a circle with radius of 60 pixels with 20 pixels spacing. SIFT descriptors are constructed in $64 \times 64$ windows placed at each landmark. Gradient magnitude at each pixel is accumulated in accordance with its orientation which is summarized into an 8-bin orientation histogram. The gradient magnitude and orientation are calculated using $M(x, y)$ as

$$
\begin{aligned}
& M_{m}(x, y)=\sqrt{\left(\frac{\partial M(x, y)}{\partial x}\right)^{2}+\left(\frac{\partial M(x, y)}{\partial y}\right)^{2}} \\
& O_{m}(x, y)=\arctan \left(\frac{\partial M(x, y)}{\partial y} / \frac{\partial M(x, y)}{\partial x}\right)
\end{aligned}
$$

The features obtained from all the sub-windows are concatenated from left to right and top to bottom to construct the final feature vector with a dimensionality of $3,712(29 \times 128)$.

The original SIFT descriptor (Lowe, 2004) construction consisted of five steps: (i) smoothing, (ii) gradient orientation \& magnitude calculation, (iii) Gaussian weighting, (iv) tri-linear interpolation, and ( $v$ ) truncation. We have empirically determined that steps $(i)$ and (iii) are redundant in generating robust feature vectors for our application. The smoothing operation is already applied in generating the gradient magnitude image, so step ( $i$ ) leads to oversmoothing, resulting in a loss of matching accuracy. We have also observed a drop in the matching accuracy after applying the Gaussian weighting, which indicates that the information contained in each sub-window is equally important. This is also partly due to the fact that, in our application, the keypoints are preset on a grid. Fig. 6 shows the schematics of tri-linear interpolation and truncation steps in the SIFT descriptor construction.
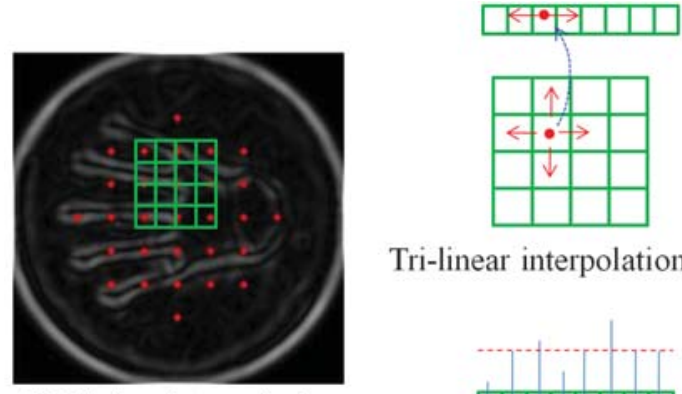

Tri-linear interpolation

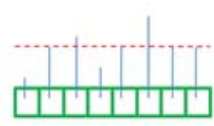

Truncation

Figure 6: Schematic of the SIFT descriptor construction. 


\subsubsection{MLBP descriptor}

Multi-scale Local Binary Pattern (MLBP) (Ojala et al., 2002) descriptor is another well known method to construct a feature descriptor. MLBP descriptor is the extended variation of the LBP descriptor by considering multiple radii and neighboring pixel sets. There is a trade-off between the matching accuracy and computational requirement in constructing the MLBP descriptor. Small values of radii and number of neighboring pixels leads to low matching accuracy, whereas large values of radii and neighboring pixels leads to high computational cost.

We used two different radii, $R=\{1,2\}$ and three different numbers of neighboring pixels, $P=\{8,4,12\}$ for effective feature vector construction. This results in six different parameter sets. MLBP histograms associated with each parameter set are constructed within each subwindow analogous to the SIFT descriptor. Histograms from different parameter sets are concatenated to form the final feature vector. Even though we only selected two different values of $R$ and three different values of $P$, this still results in a large feature vector with a dimensionality of 3,344 for each sub-window. To avoid the curse of dimensionality, we down sample the feature vectors by grouping every 16 consecutive feature values and taking the summation (reduction factor of 1/16). The final lengths of SIFT and MLBP descriptors are 3,712 and 6,061, respectively. Figs. 7 shows the parameter sets that we used for constructing the MLBP descriptors.

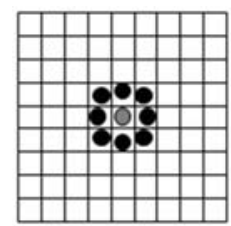

$\mathrm{P}=8, \mathrm{R}=1.0$

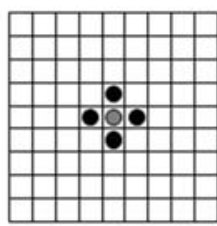

$\mathrm{P}=4, \mathrm{R}=1.0$

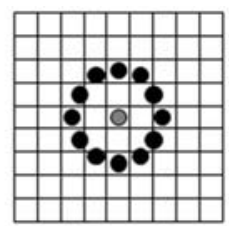

$\mathrm{P}=12, \mathrm{R}=2.0$
Figure 7: Schematic of the MLBP descriptor construction.

\subsection{Color}

The color feature vector is represented as a histogram. In general, the color histogram provides useful clues for the subsequent expression of similarity between images, due to its robustness to background complexity and object distortion (Wang et al., 2010). A three-dimensional histogram is obtained by equally dividing each of the red, green, and blue color channels (0 255) into four bins $(4 \times 4 \times 4=64$ bin histogram). The color histogram is defined as

$$
h\left[C_{R}, C_{G}, C_{B}\right]=N_{p} \cdot \operatorname{Prob}\left\{r \in C_{R}, g \in C_{G}, b \in C_{B}\right\}
$$

where $C_{R}, C_{G}$, and $C_{B}$ denote bins of each color channel, r, g, and b are the RGB color value of a pixel, respectively and $N_{p}$ is the total number of pixels in the image (Smith and Chang, 1996). To calculate the similarity between two color histograms, we compute their correlation using the Bhattacharyya coefficient (Bhattacharyya, 1943), defined as

$$
B C(p, q)=\sum_{x} \sqrt{p(x) q(x)}
$$

where $p(x)$ and $q(x)$ are the discrete color histograms.

\subsection{Shape}

We extract the invariant moments only from the pill boundary shape because the shape of imprints are encoded in the SIFT and MLBP based descriptors. From the gradient magnitude image, we extract the most significant outer boundary of the pill and use a set of moment invariants introduced by $\mathrm{Hu}(\mathrm{Hu}, 1962)$ to construct the shape feature vector.

Given the gradient magnitude image $M(x, y)$, the central moment of order $(a+b)$ is defined as

$$
\mu_{a, b}=\sum_{(x, y)}(x-\bar{x})^{a}(y-\bar{y})^{b} M(x, y)
$$

where $(\bar{x}, \bar{y})$ is the center of the image. Scale invariant moments $\eta_{i, j}$, where $(i+j) \geq 2$ can be constructed by using the following formula.

$$
\eta_{i, j}=\frac{\mu_{i, j}}{\mu_{0,0}^{\left(1+\frac{i+j}{2}\right)}}
$$

Based on the $2^{\text {nd }}$ and $3^{\text {rd }}$ order moments $((i+j)=2,3)$, a set of seven features are obtained ( $\mathrm{Hu}, 1962)$. The features are derived from algebraic invariants applied to the moment generating function under a rotation transformation. The result is a set of seven moment invariants, which can be used for scale, position, and rotation invariant pattern identification.

\subsection{Rotation normalization}

Given a gradient magnitude image, $M(x, y)$, we calculate the gradient orientation at each pixel (Eq. 3) and construct a 36-bin orientation histogram. Similar to the major orientation decision scheme for each keypoint in SIFT, we first select the most dominant orientation. Then, all other orientations within $80 \%$ of the most dominant orientation value are also selected as major orientations. For each major orientation, the gradient magnitude image is rotated, so that each major orientation aligns with the leftmost side of each pill image. 
This results in enrolling multiple templates for a pill, one corresponding to each major orientation. All these templates are stored in the database and used during the matching stage. Number of major orientations obtained for pill images in our database varies from 1 to 17. Since the invariant moments and color values are rotation invariant, we apply this rotation normalization process only to the imprint descriptor construction.

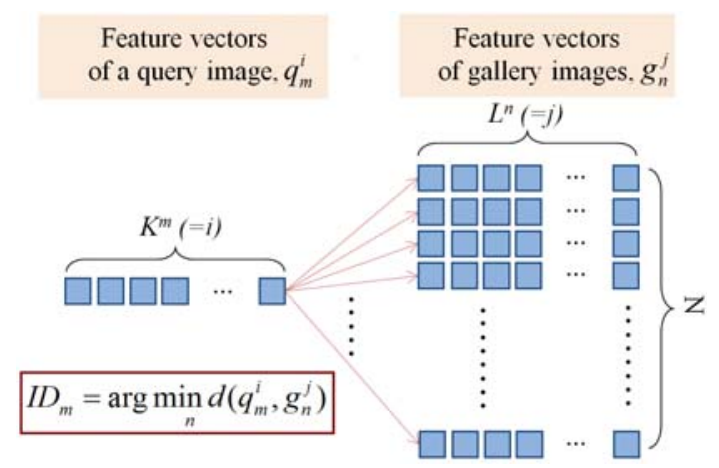

Figure 8: A schematic of the proposed matching scheme using multiple templates.

\subsection{Matching}

Since we generate multiple templates from each image to handle rotation variations ${ }^{2}$, we need to evaluate a large number of matchings. Suppose $K^{m}$ is the number of templates of the $m^{\text {th }}$ query image and $L^{n}$ is the number of templates of the $n^{\text {th }}$ gallery image. The closest match of the $m^{\text {th }}$ query in the gallery is selected as

$$
I D_{m}=\arg \min _{n} d\left(q_{m}^{i}, g_{n}^{j}\right)
$$

where $n=1, \ldots, N, i=1, \ldots, K^{m}, j=1, \ldots, L^{n}$ and $d(.,$.$) is the distance metric based on the L_{2}$-norm. The $I D(=n)$ of the closest gallery image is assigned as the $I D$ of the query (see Fig. 8). We calculate the matching accuracy using individual feature descriptors as well as their combinations. Min-max normalization and weighted sum method are used for the fusion (Jain et al., 2005). The final matching accuracy is evaluated using four different strategies: (i) using pill imprint only, (ii) using pill imprint and color, and (iii) using pill imprint, color, and shape.

\footnotetext{
${ }^{2}$ Even though we use rotation invariant shape moments, it is not very effective in differentiating pill images as was shown in (Lee et al., 2010). Therefore, we explicitly normalize the rotation variation using the major gradient orientations in each pill image.
}

\section{Experimental results}

\subsection{Database}

We received 891 illicit drug pill images from the Australian Federal Police. We also downloaded 138 illicit drug pill images and 14,002 legal pill images from the following web sites: U.S. Drug Enforcement Administration's Office of Forensic Sciences (DEA), Drug information online $\mathrm{v}^{\mathrm{v}}$, and pharmer.org ${ }^{\text {viii }}$. Thus, we have a total of 15,031 pill images. The image size of the pill varies from $48 \times 42$ to $2,088 \times 1,550$ (width $\times$ height) with 96 dpi resolution.

To evaluate the Pill-ID system, we first identified duplicate images in our database. Duplicate images in the database imply that the same type of pill was imaged two or more times (in the case of illicit drugs, it means the same illicit pill was seized several times and recorded in the database). To establish a ground truth for computing the retrieval accuracy, we assigned a unique identification number to each pill image based on imprint, shape of pill boundary, and color information. Pill images that have the same imprint, pill boundary shape, and color are assigned the same ID.

We used Munsell color system (Wyszecki and Stiles, 1967) to assign color labels. The color space is divided into 10 categories (red, yellow-red, yellow, greenyellow, green, blue-green, blue, purple-blue, purple, red-purple) and into 7 categories for gray levels. Wide usage of Munsell color system by American National Standard Institute (ANSI) and United States Geological Survey (USGS) shows its effectiveness.

Shape is also labeled in a similar manner as color with representative shape primitives (e.g., round, ellipse, egg, barrel, rectangle, 3, 4, 5, 6, 7, 8-folded, Smurf, kidney, etc.). The total number of duplicate images identified is 7,637. We use 105 and 100 distinct patterns of illicit and legal pill images, respectively as query and the entire database as gallery. Therefore, the total number of query images is 805 (444 illicit and 361 legal images of 205 distinct patterns). Fig. 9 shows example duplicate images in our database. In case the color information is irrelevant (e.g., a color image is stored as gray scale), a retrieval process using only imprint and shape can be considered.

\subsection{Matching accuracy}

The performance of the Pill-ID system is evaluated using Cumulative Match Characteristic Curve (CMC). The CMC curves are computed based on 805 queries and 15,031 gallery images. The CMC curves of the MLBP descriptor, and SIFT descriptor, SIFT + color, and SIFT + color + shape are shown in Fig. 10. The 


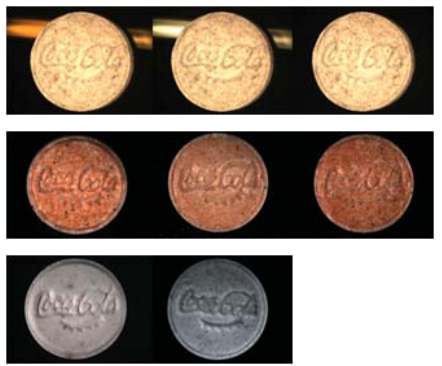

Coca-Cola

Yellow

round

Coca-Cola

Yellow-red

round

Coca-Cola

Gray03

round

Figure 9: Duplicate images in our database and their feature properties. "Coca-Cola" is the imprint name and Gray-03 represents the third category among the 7 different classes of gray color. All properties are assigned passively and used in the matching stage.

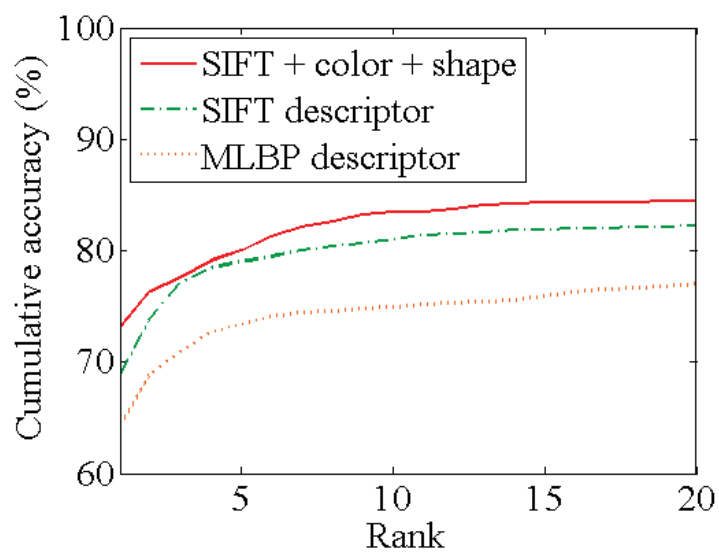

Figure 10: CMC curves showing the retrieval performance. The rank1 accuracy represents the percentage of queries whose correct mate is retrieved as the closest match.

matching scores using imprints, color, and shape are summed with empirically chosen weights for corresponding set of features under consideration to calculate the CMC curves in Fig. 10.

Example matching results for ten queries using imprint, color, and shape features are shown in Fig. 11-(a). The first five queries in Fig. 11-(a) are correctly matched at rank-1. The last five queries are not matched correctly at rank-1, but they were correctly matched in the top 20 ranks. Fig. 11-(b) shows failed matching examples in the top 20 ranks. These failures are due to the analogous imprints and colors between queries and top retrieved images and wrong segmentation. Fig. 12 shows retrieval results of four queries that failed to match at rank-1. The first two rows failed to match using imprint only, but were successfully matched using imprint and color. The queries in the third and fourth rows of Fig. 12 failed to match using imprint and color but succeeded using imprint, color, and shape.
Failed

Queries

retrievals

Successful at rank-1

retrievals
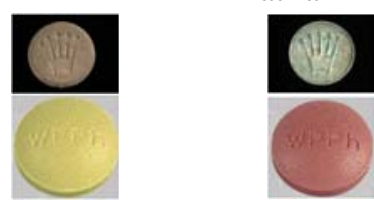

at rank-1

a) Retrievals failed using imprint alone but succeeded using both imprint and color.
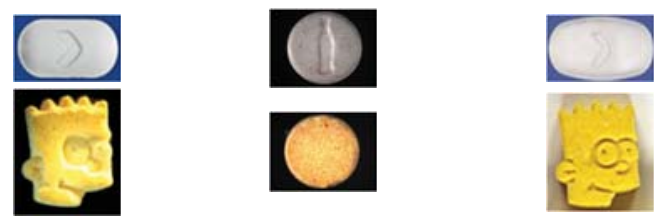

b) Retrievals failed using imprint and color but succeeded using imprint, color, and shape.

Figure 12: Retrieval examples using different combinations of features.

We make the following observations based on these experimental results: $(i)$ use of the color feature with imprint (SIFT representation) shows significant improvement compared to the use of imprint only, (ii) the Munsell color system helps to differentiate imprint patterns with different colors, and (iii) the use of pill shape marginally improved the matching performance. In law enforcement applications, it is customary to examine the top-20 retrievals. Keeping this in mind, our top-20 retrieval accuracy of about $84 \%$ is quite good.

The proposed method is implemented in Matlab on a $2.4 \mathrm{GHz}$ processor with $4 \mathrm{~GB}$ memory. The average feature extraction time based on SIFT and MLBP descriptors are 2.3 and 0.5 seconds, respectively. The retrieval time per query with $15 \mathrm{~K}$ gallery images is 13.0 and 4.0 seconds, respectively. The feature extraction and matching times for shape and color are negligible.

\section{Conclusions \& Future work}

We have developed an automatic drug pill matching and retrieval system. The system has been tested on a pill image database containing a total of 15,031 images, including 1,029 illicit drug pill images. Rank-1 (Rank-20) identification accuracy of $73.17 \%$ (84.47\%) is obtained in the matching experiments. These accuracy values are quite good, given the similarity in shape, color, and imprint information in many of the pills. This system can be used to identify illegal drug pills to assist law enforcement units by providing information such as their chemical composition, manufacturer, and manufacturing location for investigative purposes. The pro- 


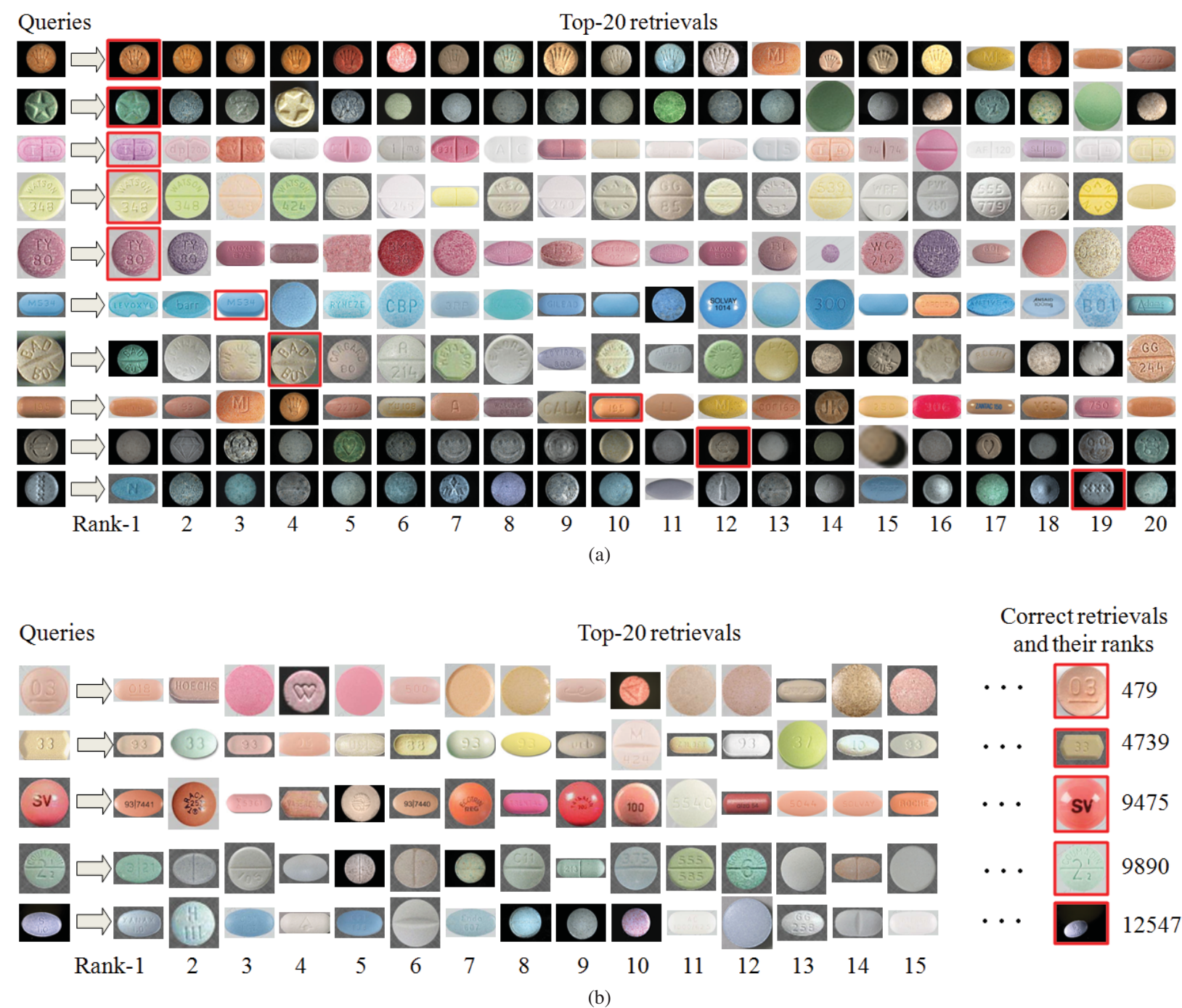

Figure 11: Example matching results using imprint, color, and shape features. (a) Correct retrievals in the top-20 retrieved images and (b) failed retrievals in the top-20 retrieved images. Correct matches are encoded with red boxes.

posed illicit drug pill identification system can also be used to identify legal drug pills or tablets when the prescription information is missing. We are improving the performance of the current system by recognizing the textual information in the imprints (digits or characters) with an Optical Character Reader (OCR). The extended method will especially help in identifying pills with missing or damaged imprints.

\section{Acknowledgment}

The authors would like to thank Dr. Mark Tahtouh of the Australian Federal Police for providing drug pill images. Anil K. Jain's research was partially supported by WCU (World Class University) program through the National Research Foundation of Korea funded by the Ministry of Education, Science and Technology (R3110008) to Korea university.

\section{URLs}

${ }^{i}$ http://www.justice.gov/ndic/pubs31/31379/index.htm ${ }^{i i}$ http://www.whitehousedrugpolicy.gov/publications/index.html

iii http://www.justice.gov/dea/programs/forensicsci/microgram/ index.html

${ }^{\text {iv }}$ http://www.accessdata.fda.gov/scripts/cdrh/cfdocs/cfcfr/ cfrsearch.cfm

${ }^{\mathrm{v}} \mathrm{http} / / /$ www.drugs.com/pill_identification.html

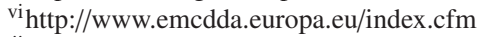

vii http://www.webmd.com/pill-identification/default.htm

viii http://www.pharmer.org/images 


\section{References}

Bhattacharyya, A., 1943. On a measure of divergence between two statistical populations defined by probability distributions. Bulletin of the Calcutta Mathematical Society 35, 99-109.

Chang, S.-K., Liu, S.-H., 1984. Picture indexing and abstraction techniques for pictorial databases. IEEE Transactions on Pattern Analysis and Machine Intelligence 6 (4), $475-484$.

Flickner, M., Sawhney, H., Niblack, W., Ashley, J., Huang, Q., Dom, B., Gorkani, M., Hafner, J., Lee, D., Petkovic, D., Steele, D., Yanker, P., Sep. 1995. Query by image and video content: the QBIC system. Computer 28 (9), 23 -32.

Geradts, Z., Bijhold, J., 2002. Content based information retrieval in forensic image databases. Journal of forensic sciences 47 (2), 285 292.

Gonzalez, R., Woods, R., 1992. Digital Image Processing (2nd ed.). Addison-Wesley Longman Publishing Co., Inc, Boston, MA.

$\mathrm{Hu}$, M.-K., 1962. Visual pattern recognition by moment invariants. IEEE Transactions on Information Theory 8 (2), 179-187.

Jain, A., Lee, J., Jin, R., Gregg, N., 2009. Content-based image retrieval: An application to tattoo images. In: 16th IEEE International Conference on Image Processing. pp. 2745-2748.

Jain, A., Nandakumar, K., Ross, A., 2005. Score normalization in multimodal biometric systems. Pattern Recognition 38 (12), 2270 -2285 .

Kato, T., 1992. Database architecture for content-based image retrieval. In: Proceedings of SPIE. Vol. 1662. p. 112.

Lee, Y.-B., Park, U., Jain, A. K., 2010. Pill-id: Matching and retrieval of drug pill imprint images. In: Proceedings of the 20th International Conference on Pattern Recognition. pp. 2632-2635.

Lowe, D., 2004. Distinctive image features from scale-invariant keypoints. International Journal of Computer Vision 60 (2), 91-110.

Ojala, T., Pietikainen, M., Maenpaa, T., 2002. Multiresolution grayscale and rotation invariant texture classification with local binary patterns. IEEE Trans. Pattern Analysis and Machine Intelligence 24 (7), 971-987.

Riesenhuber, M., Poggio, T., 2000. Models of object recognition. Nature neuroscience Supplement 3, 1199-1204.

Smith, J., Chang, S., 1996. Tools and techniques for color image retrieval. Proceedings of the SPIE Storage \& Retrieval for Image and Video Databases IV 2670, 426-437.

Wang, X., Wu, J., Yang, H., 2010. Robust image retrieval based on color histogram of local feature regions. Multimedia Tools and Applications 49 (2), 323-345.

Wyszecki, G., Stiles, W., 1967. Color Science. Wiley, New York. 